\title{
COMPUTER DISCOVERED MATHEMATICS AND APPLICATIONS IN EDUCATION
}

\author{
Sava Grozdev, Deko Dekov
}

\begin{abstract}
The computer program "Discoverer" is the first, able easily to discover new theorems in mathematics, and possibly new knowledge in science. In the present paper the authors discuss the use of "Discoverer" in teaching and learning of geometry filling a gap in the existing educational tools. "Discoverer" provides the possibility for students easily to discover new theorems in Euclidean geometry. By using the principles of "Discoverer", a number of similar programs could be created for high school teaching in: physics, chemistry, biology, and so on. Such programs could be considered as tools for interest activation. We call the use of "Discoverer" for educational purposes to be "learning through discovery", which may be considered as a new important direction within "learning through inquiry".
\end{abstract}

\section{INTRODUCTION}

An interesting information appeared recently in the Journal "New Scientist". The author of [1] asserts: "No researcher could read all the papers in their field - but machines are making discoveries in their own right by mining the scientific literature. In May 2013 a supercomputer in San Jose, California, read 100,000 research papers in 2 hours. It found completely new biology hidden in the data. Called KnIT, the computer is one of a handful of systems pushing back the frontiers of knowledge without human help. KnIT didn't read the papers like a scientist - that would have taken a lifetime. Instead, it scanned for information on a protein called p53, and a class of enzymes that can interact with it, called kinases. Also known as "the guardian of the genome", p53 suppresses tumours in humans. KnIT trawled the literature searching for links that imply undiscovered p53 kinases, which could provide routes to new cancer drugs.

2010 Mathematics Subject Classification. Primary: 51-04, 68T01, 68T99.

Key words and phrases. Feuerbach Point, Kosnita products, Haimov triangle 
Having analysed papers up until 2003, KnIT identified seven of the nine kinases discovered over the subsequent 10 years. More importantly, it also found what appeared to be two p53 kinases unknown to science. Initial lab tests confirmed the findings, although the team wants to repeat the experiment to be sure. KnIT is a collaboration between IBM and Baylor College of Medicine in Houston, Texas. It is the latest step into a weird world where autonomous machines make discoveries that are beyond scientists, simply by rifling more thoroughly through what we already know, and faster than any human can. "In general, new p53 kinases are discovered at a rate of one per year," says Olivier Lichtarge, who leads the work at Baylor. "We hope to greatly accelerate that rate of discovery." Studying kinases is important for cancer research, but the Baylor team thinks the approach can extend beyond biomedical studies to all areas of science. And if KnIT's algorithmic discoveries hold up, they point to a future in which everyone could have a personalised algorithm trawling and making sense of the scientific literature to figure out cures for their ailments, including ones tailored at a genetic level."

In [2] the following theorem is presented: If $P$ and $Q$ are isogonal conjugates with respect to triangle $A B C$, then the Ceva product of their complements lies on the Kiepert hyperbola. For the details see the cited paper. The theorem was discovered by the computer program "Discoverer", created by the authors of the present paper. The proofs of "Discoverer" are nonstandard. In [2] a proof is presented which is rewritten in a traditional manner. In [3] the authors present a list of 2364 remarkable points which lie on the Kiepert hyperbola. The list was partially produced by using the above theorem. The complete list was produced by the computer program "Discoverer".

\section{AN EXAMPLE WITH A FEURBACH POINT}

In [4] it is illustrated the elaboration of a topic from Euclidean Geometry by the computer program "Discoverer". A theorem or a group of similar theorems are used as starting points. The paper presents 283 theorems which are discovered by the computer program "Discoverer". The authors presume that some of them are new. The theorems produced by "Discoverer" could be reformulated as problems, in more than one way in the general case. Here is an example:

It is well-known, that for every point in the interior of a given angle there exist two circles through the point and touching the sides of the angle. Consider the following theorem: The Feuerbach Point is the Homothetic Center and Perspector of Triangle ABC and the Johnson Triangle of the Outer Yff Triangle. Further, consider the next 
Problem 1. Given is $\triangle A B C$. Prove, that the in-circle of $\triangle A B C$ touches the circum-circle of the medial triangle of $\triangle A B C$. Denote the common point of the circles by $F$. Let $S e$ be the outer centre of homothety of the in-centre and the circum-centre of $\triangle A B C$, while $k A\left(A_{1}, r_{A}\right)$ be the circle through $S e$, which touches the sides of $\measuredangle B A C$ in such a way, that the segment $A A_{1}$ is longer than the segment $A S e$. The circles $k B\left(B_{1}, r_{B}\right)$ and $k C\left(C_{1}, r_{C}\right)$ are defined analogously.

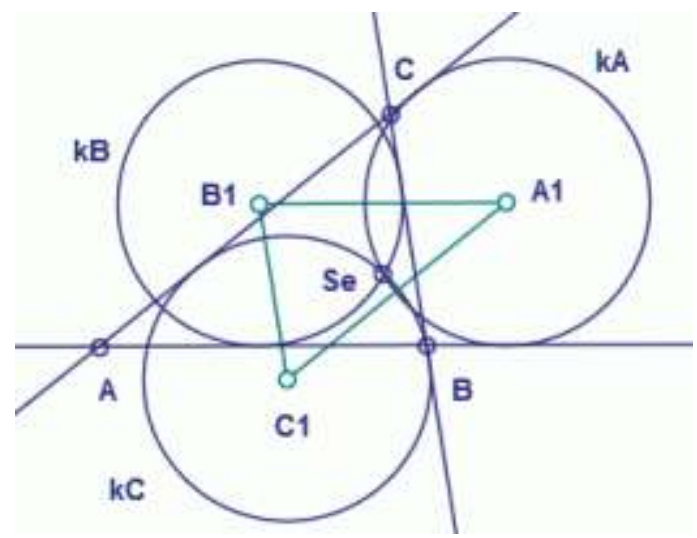

Figure 1

Let $H$ be the orthocentre of $\triangle A_{1} B_{1} C_{1}$, while $c A\left(A_{2}, r_{c A}\right)$ be the circle through the points $H, B_{1}$ and $C_{1}$. The circles $c B\left(B_{2}, r_{c B}\right)$ and $c C\left(C_{2}, r_{c C}\right)$ are defined analogously. Prove, that $\triangle A B C$ and $\triangle A_{2} B_{2} C_{2}$ are homothetic with centre of homothety $F$. Prove, that the lines $A A_{2}, B B_{2}$ and $C C_{2}$ intersect in the point $F$.

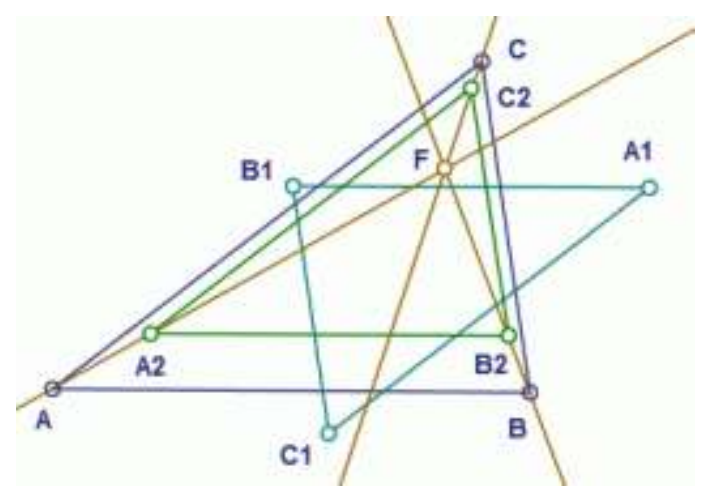

Figure 2 
The point $F$ is the well-known Feuerbach point and is denoted by $\mathrm{X}(11)$ in [5]. The circum-circle of the medial triangle of $\triangle A B C$ is the well-known Euler circle. The three circles $k A, k B$ and $k C$ are called to be outer Yff circles, while $\triangle A_{1} B_{1} C_{1}$ is called to be outer Yff triangle of $\triangle A B C$. It is well-known, that the outer Yff triangles are congruent. The circles $c A, c B$ and $c C$ are called to be Johnson circles and are congruent too. The triangle $A_{2} B_{2} C_{2}$ is called Johnson triangle.

\section{Kosnita Products}

Interesting examples are developed in [6]. In 2011 Randy Hutson introduced the notion of Kosnita products. In the encyclopedias of Weisstein [7] and Kimberling [5] some tables are given with theorems on Kosnita products. It is shown in [6] how these tables could be extended by means of the computer program "Discoverer". The paper contains 70 theorems on Kosnita products, produced by "Discoverer". The example is connected with the following theorem: The complement of the complement of the internal centre of similitude of the in-circle and the circum-circle is the perspector and homothetic centre of the medial triangle and the triangle of the circum-centres of the pedal corner triangles of the internal centre of similitude of the in-circle and the circumcircle. The theorem could be pre-formulated in the following way:

Problem 2. Given is $\triangle A B C$ and let $\triangle A_{1} B_{1} C_{1}$ be its medial triangle. Lert $S i$ be the inner centre of homothety of the in-centre and the circum-centre of $\triangle A B C$, while $\triangle A_{2} B_{2} C_{2}$ be the pedal triangle of $S i$. Denote the circum-centre of $\triangle A C_{2} B_{2}$ by $A_{3}$. The points $B_{3}$ and $C_{3}$ are defined analogously. Let $G$ be the gravity centre of $\triangle A B C$ and let $P$ divide the segment $S i G$ in inner proportion $S i P: P G=3: 1$. Prove, that the lines $A_{1} A_{3}, B_{1} B_{3}$ and $C_{1} C_{3}$ intersect in the point $P$.

Using "Discoverer" in a similar way to A1 and A2 from the figure one could examine the remaining supposing new points from the List D [7]. Note, that the List D contains 31 points, two of them coinciding. Thus, in fact the list contains 30 "new" different points. Using "Discoverer" one could identify the coinciding points. 


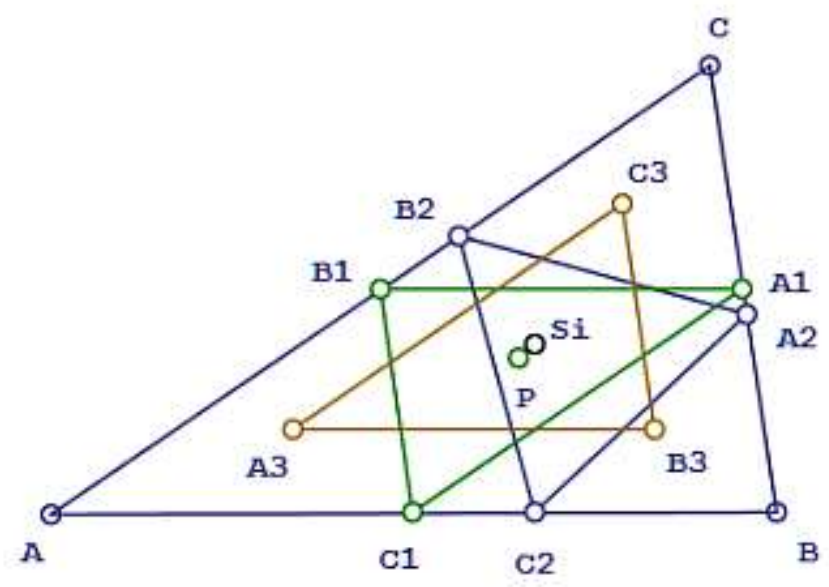

Figure 3

\section{OTHER EXAMPLES}

In [8] the authors present some results concerning Euler products and Euler transforms, all of them discovered by the computer program "Discoverer". Similarly, in [9] some results related to the Haimov triangle are presented, also generated by "Discoverer". In [10] a detailed description is given for an improvement of the classical Steiner's solution of the construction of the Malfatti circles, discovered by the computer program "Discoverer". The theory of the complexity of the geometric constructions is applied in order to obtain a numerical measure of the complexity of the solutions. The Malfatti problem is as follows: Within a given triangle draw three circles each of which is tangent to the other two and to two sides of the triangle. Given $\triangle A B C$, that is, given points $A, B$ and $C$ and lines $B C, C A$ and $A B$. The Steiner's construction has the following stages:

Stage 1. Construct the internal angle bisectors and the in-centre of $\triangle A B C$.

Stage 2. Construct the vertices of the de Villiers triangle.

Stage 3. Construct the Malfatti-Steiner point.

Stage 4. Construct the Malfatti central triangle.

Stage 5. Construct the Malfatti circles.

The steps of the construction are given in [10]. Using the Lazarov-Tabov measure (see [10]), the measure of stage 4 of the Steiner's construction is 50, while the improved by "Discoverer" stage 4 has measure 12. The Labelle's (see [10]) measure of stage 4 of Steiner's construction is 12 , while the improved by "Discoverer" stage 4 has measure 3. The complexity of the improved by 
"Discoverer" stage 4 is in the first case $24 \%$, and in the second case $25 \%$ of the complexity of the Steiner's stage 4. Hence, the computer program "Discoverer" has discovered an essential improvement of stage 4 of the Steiner's construction of the Malfatti circles.

Consider the following theorem (see [11]): Given $\triangle A B C$. Let $P$ and $U$ be points different from the vertices of $\triangle A B C$. Let $L_{1}$ be the reflection of the line $P U$ on the line $B C$, and define $L_{2}$ and $L_{3}$ cyclically. Let $D=L_{2} \cap L_{3}$, $E=L_{3} \cap L_{1}$, and $F=L_{1} \cap L_{2}$. The the lines $A D, B E$ and $C F$ concur in a point, which lies on the circum-circle of $\triangle A B C$. The construction in the theorem is published by T. Lalesco in 1937 and reprinted in 1987. We call the Lalesco product of $P$ and $U$ the point of concurrence, defined in the theorem, and we denote the Lalesco product of $P$ and $U$ by $L P(P, U)$. Note that the Lalesco product is commutative. Also, if point $V$ lies on the line $P U$, then $L P(P, V)=$ $L P(V, U)=L P(P, U)$. The next theorem is discovered by the computer program "Discoverer": Given $\triangle A B C$. Let $P$ and $U$ be points different from the vertices of $\triangle A B C$ and having barycentric coordinates $P=(p, q, r)$ and $U=(u, v, w)$. The Lalesco product $L P(P, U)$ has barycentric coordinates

$$
\begin{gathered}
(f(a, b, c, p, q, r, u, v, w), f(b, c, a, q, r, p, v, w, u), \\
f(c, a, b, r, p, q, w, u, v))
\end{gathered}
$$

where

$$
\begin{aligned}
& f(a, b, c, p, q, r, u, v, w)=a^{2}\left(q c^{2} w-b^{2} q w+q u b^{2}+q u c^{2}-a^{2} q u\right. \\
& \left.-a^{2} q w-2 p b^{2} w+b^{2} v r-v p c^{2}+a^{2} v p+a^{2} v r+2 u b^{2} r-v c^{2} r-v p b^{2}\right) \\
& \left(q c^{2} w+a^{2} q w-b^{2} q w+2 q u c^{2}-p b^{2} w-p c^{2} w+a^{2} p w\right. \\
& \left.-a^{2} v r+b^{2} v r+u c^{2} r-a^{2} u r-2 v p c^{2}+u b^{2} r-v c^{2} r\right) .
\end{aligned}
$$

By using the computer program "Discoverer" the Cevian corner products are investigated in [12]. The paper contains more than 1000 theorems. Similarly, more than 30 theorems are proposed in [13] about antipedal corner products, while the paper [14] contains more than 100 theorems about pedal corner products, The expectations are, that the majority of these theorems are new, discovered by a computer.

\section{CONClusion}

The computer program "Discoverer" is an artificial intelligence system for discovery of new knowledge, able easily to produce new theorem in Euclidean geometry. As far as the authors know, the "Discoverer" is the first computer 
program, able easily to discover new theorems in mathematics, and possibly, the first computer program, able easily to discover new knowledge in science. The use of "Discoverer" now begins, so that we could expect many remarkable results in the future.

References

[1] H. Hodson, Automated discovery, New Scientist, 2984 (223), (2014), 19-20

[2] S. Grozdev, D. Dekov, Computer generated mathematics: points on the Kiepert hyperbola, The Mathematical Gazette, note 98.33, (2014), 37-38

[3] S. Grozdev, D. Dekov, Points on the Kiepert Hyperbola, Journal of Computer-Generated Mathematics, 2 (8), (2013) http://www.ddekov.eu/j/contents.htm\#2013

[4] S. Grozdev, D. Dekov, Computer generated mathematics: Kosnita products in Euclidean geometry, Mathematics and Informatics, 4 (57), (2014), 34-42

[5] C. Kimberling, Kimberling's Encyclopedia of Triangle Centers, http://faculty.evansville.edu/ck6/encyclopedia/ETC.html

[6] S. Grozdev, D. Dekov, Computer generated mathematics: Kosnita products in Euclidean geometry, Mathematics and Informatics, 4 (57), (2014), 355363

[7] E. W. Weisstein, MathWorld - A Wolfram Web Resource. http://mathworld.wolfram.com/

[8] S. Grozdev, D. Dekov, Machine approach to Euclidean geometry: Ueler triangles, Euler products and Euler transforms, Mathematics and Informatics, 5 (57), (2014), 519-528

[9] S. Grozdev, D. Dekov, Computer generated mathematics: a note on the Haimov triangle, Mathematics and Informatics, 6 (57), (2014), 559-567

[10] S. Grozdev, D. Dekov, The computer improves the Steiner's of the Malfatti circles, Mathematics and Informatics, 1 (58), (2015), 40-51

[11] S. Grozdev, D. Dekov, Computer generated mathematics: Lalesco products, Mathematics and Informatics, 2 (58), (2015), 143-148

[12] S. Grozdev, D. Dekov, Computer generated mathematics: Cevian corner products, Mathematics and Informatics, 4 (58), (2015), 426-436

[13] S. Grozdev, D. Dekov, Computer generated mathematics: antipedal corner products, Mathematics and Informatics, 5 (58), (2015), 513-519

[14] S. Grozdev, D. Dekov, Computer generated mathematics: pedal corner products, Mathematics and Informatics, 6 (58), (2015), 609-615 
${ }^{1)}$ VUZF University, Sofia, Bulgaria

E-mail address: sava.grozdev@gmail.com

${ }^{2)}$ Stara Zagora, Bulgaria

E-mail address: ddekov@ddekov.eu 